\title{
INCORPORACIÓN DE LAS TIC POR PARTE DE PROFESORES UNIVERSITARIOS COLOMBIANOS
}

\section{INCORPORATION OF TIC (INFORMATION AND COMMUNICATION TECHNOLOGY) TO COLOMBIAN UNIVERSITY TEACHERS}

\author{
Esp. Héverd Augusto Páez Quintana ${ }^{\text {, }}$, Maricarmen Cantú Valadez , Ph.D. Catalina Rodríguez \\ Pichardo ${ }^{\mathrm{c}}$
}

\author{
${ }^{a}$ Universidad Francisco de Paula Santander Ocaña, Grupo de Investigación GIFEAH, Vía \\ Acolsure Sede el Algodonal, Ocaña - Norte de Santander, Colombia, hapaezq@ufpso.edu.co \\ ${ }^{b}$ Tecnológico de Monterrey. Docente en el sistema de educación normal en Nuevo León, \\ México. Profesora de cátedra en el en la línea de investigación de competencias integrales \\ educativas. maricarmen.cantu@itesm.mx \\ ${ }^{\mathrm{c}}$ Profesora-investigadora del ITESM y dictaminadora en Revistas científicas, miembro de IAAP \\ e IAVEG. cmrodrig@itesm.mx
}

\begin{abstract}
Resumen: Este trabajo presenta una investigación sobre la aplicación de las TIC de los profesores colombianos, reflejando la experiencia de los docentes de la carrera de Comunicación Social de la Universidad Francisco de Paula Santander Ocaña. El método seleccionado fue cualitativo descriptivo. Los resultados obtenidos se dieron a través de la aplicación de una entrevista semi-estructurada dirigida a cuatro docentes de las cátedras: Investigación Cuantitativa, Proyecto de Vida, Expresión y diseño gráfico y Prensa, Como también se realizó la observación no participativa de la intervención y aportaciones de 15 estudiantes de las asignaturas escogidas. La principal conclusión es que las competencias de los estudiantes en TIC están relacionadas al modelo micropedagógico de las asignaturas y al aporte que cada docente le ha implementado en el proceso de enseñanza-aprendizaje en el aula. De ahí la importancia que el programa académico incorpore un nuevo modelo micropedagógico con un componente claro de las competencias que deben desarrollar los estudiantes apoyados en las TIC como eje transversal al proceso de formación de los comunicadores sociales.
\end{abstract}

Palabras clave: Competencias, Micropedagógico, didáctica.

Abstract: This work presents an investigation of the application of TIC to Colombian teachers, reflecting the experience of teachers of the Social Communication program at University Francisco de Paula Santander Ocaña. The selected method was the qualitative descriptive. The 
outcome was obtained through the application of a semi-structured interview addressed to four teachers of the following lectures: Quantitative research, Life Project, Expression and Graphic design, and press. Besides, it was made a non-participative observation of the intervention and the contribution of 15 students of the chosen subjects. The main conclusion is that the competences of the students who use the ICT are related to the micro-pedagogical model of the subjects and the teachers have contributed to the implementation during the teaching-learning process in the classroom. Therefore, it is essential that the academic program incorporates a new micro-pedagogical model with a clear component of the skills that the students need to develop supported by TIC as a cross-cutting element in their formation process in social communication.

Keywords: TIC, Micro Pedagogy, Didactic.

\section{INTRODUCCIÓN}

El proyecto Tuning originado en el Marco Común Europeo, ofrece la posibilidad de examinar las competencias genéricas y específicas buscando alinear la titulación y la formación profesional bajo la fórmula de Crédito Europeo. Sánchez y García (2007), hacen referencia a las competencias en Tecnología Educativa enmarcada en la formación de los docentes y a su vez el conocimiento que debe recibir el educando. Entre las competencias se determinan:

Profundizar en el conocimiento del diseño de la enseñanza a partir de diferentes concepciones curriculares.

Desarrollar una capacidad creativa aplicada en relación al diseño de materiales educativos.

Iniciarse en los conceptos básicos referidos a los multimedia en sus aplicaciones educativas.

Analizar diferentes modelos evaluativos para la validación de medios y materiales educativos.

Este panorama empata con el proyecto Tuning para América Latina, donde se tomó el modelo europeo buscando alinear el proceso de internacionalización de la educación y siguiendo su propia metodología en cuatro grandes líneas: competencias (genéricas y específicas de las áreas temáticas); enfoques de enseñanza, aprendizaje y evaluación; créditos académicos; y calidad de los programas.

Este proceso originó la elaboración de una lista de 30 competencias clasificadas en tres grupos: instrumentales, interpersonales y sistémicas, relacionadas con la capacidad del educando para desarrollarse y las competencias de los docentes Sánchez y García (2007), de las cuales se destacan algunas:

- Compromiso con el progreso y rendimiento del alumno.

- Conocimiento y dominio de diferentes estrategias de enseñanza-aprendizaje.

- Conocimiento de la materia a enseñar.

- Capacidad para crear un clima apropiado y favorecedor del aprendizaje. 
- Capacidad para utilizar las TIC en integrarlas en entornos de aprendizaje.

- Capacidad para gestionar el tiempo eficazmente.

- Capacidad para reflexionar sobre la propia actuación y autoevaluarse.

- Toma de conciencia de la necesidad de desarrollo profesional continuo.

- Capacidad para evaluar los resultados del aprendizaje y el rendimiento de los alumnos.

- Capacidad para resolver problemas de forma colaborativa.

- Capacidad para responder a la diversidad del alumnado.

- Capacidad para mejorar el entorno de enseñanza-aprendizaje.

- Capacidad para adaptar el currículum.

\section{Competencias en TIC en el entorno Universitario}

Guevara (2012), en la investigación que realizó sobre evaluación de competencias en educación superior, define el término en su acepción etimológica, que proviene del latín compêtere, cuyo significado es: aspirar, ir al encuentro de... Este término da lugar a los verbos competer, incumbir, pertenecer.

Muchos autores han conceptuado sobre competencias. El concepto se unifica al establecer que las competencias profesionales están circunscritas a un conjunto identificable y evaluable de capacidades (conocimientos, habilidades y actitudes). Para tener una definición más clara, García, Loredo, Luna, Rueda (2008) señalan que el modelo ECD se plantea que: Tanto en las competencias profesionales como en las laborales, se distinguen las genéricas (generales o "transversales"), que son las comunes a todas las profesiones u ocupaciones y constituyen en su conjunto el perfil profesional de un egresado. Por su parte, las competencias específicas o particulares son las que distinguen a cada una de las profesiones u ocupaciones del resto.

La formulación para unificar las competencias, dieron auge a otros modelos de aprendizaje como el electronic, blended y mobile learning, que migraron a la educación tradicional buscando abrir espacios y cerrar brechas. Partieron desde la educación presencial y multimodal, para mundo.

Ramírez (2012) hace énfasis, en ese sentido, que los modelos y estrategias de enseñanza para ambientes innovadores han venido evolucionando para convertirse en innovación para los sistemas y el currículo, así como cambiaron las técnicas, los recursos, el docente, el estudiante y el sistema.

Las instituciones de educación superior asumieron el desarrollo tecnológico como el motor para modificar las formas tradicionales de enseñanza para pasar a modelos curriculares más abiertos y flexibles que interactúen con la enseñanzaaprendizaje dentro y fuera del aula con un componente transversal que son las TIC.

La formación de estudiantes en competencias mediadas por las TIC, obliga a reflexionar y construir micropedagógicos que permitan que el proceso tenga todos los componentes: institucionales, docencia, investigación, extensión, responsabilidad 
social así como la participación activa y comprometida del educando. Sumado a ello el estudio permanente de las necesidades del contexto para responder efectivamente.

Este contexto se evidencia en el entorno universitario colombiano, donde los currículos han sido modificados buscando mayor acercamiento al desarrollo de competencias en todas las áreas, una de ellas en TIC, donde el docente debe estar preparado para enfrentar los cambios permanentes en las tecnologías que permiten mejorar las condiciones del aula y mejorar las capacidades del educando.

\section{Competencias en TIC}

Los estudios realizados alrededor del mundo, especialmente en Europa, reflejan la importancia del docente y las competencias que debe tener para formar al educando. Peralta y Costata (2007), en su investigación sobre los problemas surgidos para enseñar con eficacia con las TIC, hacen referencia a que: los profesores son una de las principales dificultades para introducir innovación en la educación. Muchos ni siquiera están preocupados el enfoque de aprendizaje que siguen... Los profesores no son conscientes de su plan de estudios individualizado ni están preocupados con los enfoques constructivistas del aprendizaje y la utilización de las TIC para enfatizar metodologías abiertas, procedimientos de trabajo, investigación y autonomía del estudiante.

Preocupados por la formación de los docentes en TIC, la Unesco (2008) en su propuesta para definir las competencias que deben tener los profesores, lo explica de manera explícita: Hoy en día, los docentes en ejercicio necesitan estar preparados para ofrecer a sus estudiantes oportunidades de aprendizaje apoyadas en las TIC; para utilizarlas y para saber cómo éstas pueden contribuir al aprendizaje de los estudiantes, capacidades que actualmente forman parte integral del catálogo de competencias profesionales básicas de un docente. Los docentes necesitan estar preparados para empoderar a los estudiantes con las ventajas que les aportan las TIC.

En ese sentido, Shawki, T. (2008) establece que las competencias en TIC de los docentes deben estar enfocadas en 1) Nociones Básicas de TIC: Consiste en preparar estudiantes, ciudadanos y trabajadores, para que sean capaces de comprender las nuevas tecnologías (TIC) y puedan así apoyar el desarrollo social y mejorar la productividad económica.

Continuando con 2) la Profundización del Conocimiento: Consiste en incrementar la capacidad de estudiantes, ciudadanos y trabajadores para agregar valor a la sociedad y a la economía, aplicando conocimientos de las disciplinas escolares a fin de resolver problemas complejos y prioritarios con los que se encuentran en situaciones reales en el trabajo, la sociedad y la vida.

Para terminar en 3) Generación de Conocimiento: Consiste en incrementar la productividad, formando estudiantes, ciudadanos y trabajadores que se comprometan continuamente con la tarea de generar conocimiento, innovar y aprender a lo largo de toda la vida y que se beneficien tanto de la creación de este conocimiento como de la innovación y del aprendizaje permanente.

Bajo estas premisas, debe considerarse que el educador debe tener capacidad de innovar, encontrar soluciones creativas y 
adaptarse al cambio. Un docente es quien ha desarrollado competencias para el desempeño ciudadano, es una persona que tiene conocimiento y los aplica, quien está motivado para aprender de forma continua a lo largo de la vida (Tobón, 2006). Es decir, el docente necesita desarrollarse como persona para luego tener las competencias profesionales o específicas.

El docente debe cambiar de rol y propiciar que el proceso se base en el estudiante, donde el educador cumple la función de coordinador, acompañante, guía, asesor y orientador de tal proceso. El verdadero protagonista es el estudiante, quien debe ser artífice de su aprendizaje. Para lograr el objetivo el docente debe abrir espacios y cambiar de mentalidad, el enfoque didáctico, el sistema de evaluación y la estrategia de enseñanza (Tolosana, 1990).

Así lo ratifica Díaz (2006), cuando indica que la formación del docente en la mayoría de los casos, concluye en la escolaridad al no contar con un plan de formación permanente. Es importante que la formación del docente de la UFPS Ocaña esté fundamentado en lo que establece el modelo pedagógico adoptado por la Institución desde el Constructivismo Social donde hace énfasis en la función del docente como el facilitador y dinamizador de los procesos formativos.

\section{Las TIC como medio para desarrollar las competencias}

Como lo indica Sancho (2008) la teoría sicológica cultural de Vigotsky, constituye un marco de referencia ideal para desarrollar y comprender los procesos de transformación social generados por las TIC. Para Vigotsky (1988) la educación, no solo implica el desarrollo potencial del individuo sino también la expresión y el crecimiento histórico de la cultura humana de las que surge el hombre; en este sentido, se puede indicar que las TIC permiten desarrollar las competencias en el educando a partir de su interacción con otras culturas.

Varios conceptos se expresan al determinar la mediación cognitiva como un proceso de desarrollo del pensamiento superior. Para Baquero (1997) la cognición del estudiante se media a través de sus habilidades psicológicas y se construye a través de la interacción con los demás, mediados por la cultura.

Coll (2008) asegura que toda actividad humana está mediada por el uso de herramientas y el desarrollo cognitivo ocurre por la apropiación de las herramientas (simbólicas o materiales) del plano cultural en las cuales se halla incluido el estudiante. Y enfatiza el autor en que las TIC son transformadoras, definen los procesos evolutivos de los seres humanos, desarrollan habilidades motrices y cognitivas, crean percepciones, comprensiones del mundo, relaciones sociales, prácticas y comportamientos culturales acordes con su apropiación y uso. Este desarrollo de la tecnología, opera en la zona de desarrollo próximo a partir de la internalización de las habilidades cognitivas requeridas por los sistemas de herramientas y promoviendo prácticas acordes a su manera de pensar (Coll, 2008).

Esas habilidades se desarrollan a partir del tipo y el uso de las TIC, que se clasifican en todas las herramientas de las que se dispone en el aula para modernizar las prácticas educativas y que marcan un rompimiento con la convencionalidad del docente que explica un tema frente al tablero. Aquí se incluyen, entre otras, 
proyectores, pizarras interactivas, Software multimedia, sistemas para la gestión del aprendizaje (Learning management system, LMS) y herramientas Web 2.0.

El Estado Colombiano incluyó la competencias en TIC en los Proyectos Educativos de educación básica, media y superior, MEN (2013). Sin embargo su uso y aprovechamiento todavía no alcanzan los resultados esperados. Así lo asegura Llinás (2000) cuando sugirió al gobierno nacional, que gran parte del aumento en competencias para el desarrollo científico es transversal al aumento de docentes formado en TIC. El científico hace referencia al manejo y diseño de ambientes de aprendizaje mediados por las nuevas tecnologías.

Estas recomendaciones, aseguran los autores (Coll, 2008, Sancho, 2008, Navarro, 2008, Hargreaves, 2003) buscan mejorar las competencias, la motivación y las capacidades cognitivas del estudiante, que permitan la diversificación de códigos de representación de la información, al facilitar la inserción de diversos lenguajes, posibilitan el paso de la sociedad de la Información a la Sociedad del Conocimiento, promueven el aprendizaje autónomo, propician el aprendizaje por intercambio y cooperación, presentan alternativas para asumir riesgos, formulan hipótesis, reconocen al otro y sus diferencias, generando con todo esto, oportunidades para fortalecer los diferentes estilos de aprendizaje de los sujetos involucrados en el proceso de enseñanza aprendizaje.

Para alcanzar estos propósitos del uso y apropiación de las TIC en los estudiantes de Comunicación Social de la Universidad Francisco de Paula Santander Ocaña, se buscó generar una reflexión entre docentes y estudiantes sobre la tecnología, el desarrollo de competencias y conllevar a incluir estrategias de enseñanzaaprendizaje innovadoras en la reforma curricular y establecer la Didáctica como eje central. (Acuerdo 006, 2003).

\section{La didáctica como eje para el desarrollo de competencias}

Para Zambrano (2002) la didáctica es la disciplina a través de la cual se reflexiona sobre las condiciones en las que se dan la producción, circulación y apropiación del conocimiento, así cada área del conocimiento o disciplina requiere el fortalecimiento de didácticas que se adecúen a sus necesidades particulares, pues su finalidad es desarrollar el cómo se hace, la manera y el método que le permite a un individuo apropiarse del conocimiento.

Para el profesor (Tobón, 2006), la didáctica implica la reflexión sobre la enseñanza, sobre el diseño del material utilizado para educar, y estudia la construcción de los dispositivos didácticos -estrategias de enseñanza aprendizajeidóneos para el aprendizaje. Se define como la secuencia de etapas que se ejecutan para lograr unos determinados objetivos, a través de la regulación y optimización de los procesos afectivos, cognitivos y psicomotrices; constituyen actividades conscientes y deliberadas que requieren planificación y control, e implican la selección de recursos cognitivos. Son planes dirigidos a metas, se ponen en marcha a partir de la iniciativa de los estudiantes, y se relacionan con el procesamiento de la información en torno a actividades y problemas, buscando el desempeño idóneo. 
Esa didáctica según Salinas, J. (2004), sirve como un orientador y la escogencia de elementos necesarios en los entornos virtuales de formación para entender un modelo de formación flexible y darle mayor énfasis a la formulación de currículos consideramos los siguientes componentes: - Comunicación mediada por ordenador (Componente tecnológico) Medios didácticos (componente curricular) - Flexibilidad (Elementos del aprendizaje abierto) - Entorno organizativo (Componente institucional) - Aprendizaje y tutoría (Componente didáctico).

Esta demanda de nuevas metodologías como el aprendizaje basado en la experiencia y la autonomía del estudiante, permite que el trabajo pedagógico fortalezca las competencias a partir de nuevas estrategias didácticas que deben ser integradas al micropedagógico de la carrera de Comunicación Social de la UFPS Ocaña.

\section{Modelo Micropedagógico}

Analizando el contexto, se debe realizar una indagación sobre la aplicación de las TIC en el sistema micropedagógico de la carrera de Comunicación Social a partir de la aplicación de instrumentos pertinentes para determinar el uso de las TIC por parte de los docentes en cuatro asignaturas: Investigación Cuantitativa, Proyecto de Vida, Expresión y diseño gráfico y Prensa. Esta investigación resulta importante para determinar cuáles serían las transformaciones que deben surgir a nivel micropedagógico para encontrar puntos de encuentro que permitan que las TIC ayuden al desarrollo curricular y por ende al perfil profesional que se busca del comunicador social de la Universidad Francisco de Paula Santander Ocaña.
Todo ello es importante para realizar una indagación sobre cómo aplican las TIC los docentes mediante una entrevista semiestructurada y los estudiantes de las cuatro asignaturas a través de una observación, partiendo de la siguiente pregunta de investigación:

¿De qué manera incorporan las TIC algunos profesores y estudiantes colombianos de una universidad pública en 4 clases (Investigación Cuantitativa, Proyecto de Vida, Expresión y diseño gráfico y Prensa) como referente de insumo en la construcción de un modelo micropedagógico?

\section{METODOLOGÍA}

La investigación busca identificar y analizar el uso y aprovechamiento de las TIC por parte de los docentes y estudiantes universitarios, en particular de cuatro asignaturas de la carrera de Comunicación Social de la Universidad Francisco de Paula Santander Ocaña. Para Hernández, Fernández, \& Baptista (2003), dicho comportamiento como cualitativo descriptivo, busca reconstruir la realidad tal y como la observan los actores de un sistema social previamente definido, por tanto esta investigación pretende identificar las competencias en TIC de los docentes y los estudiantes con el enfoque seleccionado.

El tipo de estudio de la investigación es descriptivo, ya que se orienta a la identificación de las competencias en TIC de los docentes en el aula de clase y su orientación a los estudiantes de la carrera de Comunicación Social buscando que, mediante el método deductivo, conseguir verdades particulares y convertirlas en evidentes. 
Se desarrollaron y aplicaron las técnicas de recolección de datos como la observación, donde, mediante una rejilla de observación, permitió al investigador apoyarse para lograr obtener mayor número de datos al describir las actividades, conductas y acciones que desarrollan los participantes en escenarios comunes como el aula de clase (sala de informática), y que de otra manera sería difícil de obtener. La rejilla de observación no participativa se limitó a registrar la información que desarrollaban los estudiantes.

La entrevista busca que de manera clara los docentes determinen si las prácticas en TIC que mencionan están relacionadas con las descritas en el micropedagógico de la asignatura y cómo se lleva a cabo en el aula. El instrumento desarrollado se aplicó a cuatro docentes que hacen parte del programa académico de Comunicación Social del área de humanidades.

El método buscó que los participantes tuvieran la posibilidad libre, abierta y consciente de abordar los instrumentos para obtener la mayor información fidedigna que permitiera el desarrollo de una nueva propuesta micropedagógica que mejore las competencias en TIC.

\section{MUESTRA}

El universo de estudiantes de la carrera de Comunicación Social de la UFPS Ocaña, es de 513 estudiantes de todos los semestres académicos. La muestra son estudiantes del tercer semestre donde se determinará si se formulan competencias en TIC y cómo está establecido en el micropedagógico desarrollado en el aula de clase. Son jóvenes en edades de 16 a 20 años, pertenecientes a los estratos uno y dos y con escasos recursos para acceder a la tecnología más avanzada.
La muestra es una población de 30 estudiantes que hacen parte del tercer semestre, se seleccionó una muestra por conveniencia de 15 estudiantes; de acuerdo a Maxwell (1996), la selección de la muestra se tuvo en cuenta el concepto de selección basada en criterios, selección deliberada con el fin de obtener la mejor calidad de la información. Los 15 estudiantes que hacen parte de esta investigación, son los que reciben clases en el tercer semestre y no están atrasados en las líneas de formación y cuatro docentes de tercer semestre que dirigen cátedras: Proyecto de vida (teórica), Investigación Cuantitativa (teórico-práctica), Prensa (teórico-práctica), Expresión y diseño gráfico (Práctica).

Los docentes fueron seleccionados de los que imparten cátedra en tercer semestre de la carrera de Comunicación Social, en las diferentes áreas del conocimiento. Del área básica se escogió los profesores que dirigen la cátedra de Proyecto de Vida y del área profesional: Expresión y diseño gráfico, Investigación Cuantitativa y Prensa.

\section{INSTRUMENTOS}

En primera instancia se tomaron las variables que se relacionan en la entrevista semiestructurada, donde se buscó dar respuesta a saberes informáticos de acuerdo a la variable del uso del computador por parte del profesor para orientar su clase en el aula. Además de la actitud del docente frente a las TIC para el desarrollo curricular y determinar la importancia que tienen las nuevas tecnologías para un nuevo modelo micropedagógico. En el caso de la observación permitió al investigador verificar las actividades que realizan los 15 estudiantes observados con respecto a la 
utilización de las TIC, para determinar los saberes propios de los alumnos y cómo influyen las TIC para el desarrollo de competencias en las cuatro asignaturas del estudio con los recursos y herramientas educativas que utilizan de forma particular o dirigida por el docente.

Cada procedimiento fue validado a través del instrumento aplicado. La observación, a través de la rejilla, permitió conocer cómo es el uso que los estudiantes hacen de las TIC para establecer con qué elementos digitales cuentan los jóvenes observados que les permitan realizar un aprendizaje autónomo o colaborativo. La entrevista semiestructurada establece que una vez los docentes diligencien el instrumento se consolida en categorías de análisis que agrupa las variables y le da paso a una subcategoría que permite establecer cuáles son la de mayor importancia y se definen para poder describir las respuestas de los entrevistados.

La información se representó en tablas en donde se exponen las categorías, subcategorías, la definición que se le da a las categorías y la descripción. Organizada esta información, se hace una descripción de las experiencias de los estudiantes bajo la óptica del investigador y realizar la interpretación y evaluación.

Con ello se hizo la validación de los instrumentos que son aplicados en la investigación bajo los principios de respeto por el trabajo de los docentes y el desempeño de los estudiantes.

Teniendo en cuenta la información recopilada en los instrumentos se procedió a identificar las categorías y las unidades de análisis como se muestra en la Figura 1.
Figura 1. Categorías y Unidades de Análisis

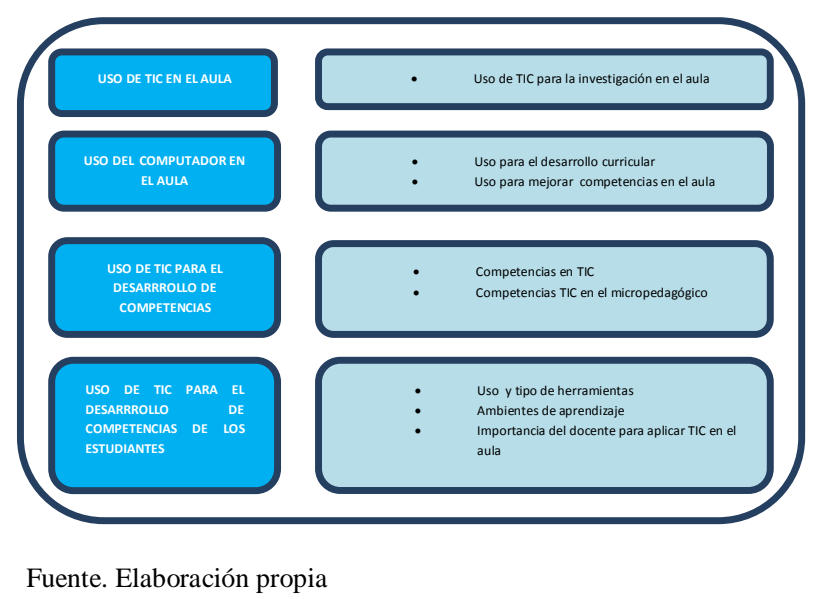

\section{PROCEDIMIENTOS}

Para la aplicación de los instrumentos se diseñó un formato de consentimiento para informarle al Director del Plan de Estudios de Comunicación Social, el director aprobó la realización de la investigación, autorizando la realización de la observación de los docentes, estudiantes y los micropedagógicos. Asimismo se le solicitó a los estudiantes la autorización para realizar la observación mientras hacían las labores propias del aula de clase.

Para la formulación y aplicación del instrumento se tuvo en cuenta:

- El diseño del instrumento se formuló de acuerdo a las variables más importantes. Para los docentes con preguntas abiertas se buscó determinar cuál es el uso que hacen de las TIC para desarrollar su asignatura como un elemento transversal a la formación del educando.

- Los instrumentos se basan en las categorías y subcategorías de análisis para hacer énfasis en las principales variables, donde se hace una definición y descripción de los hallazgos.

-Los instrumentos se aplicaron en el mes de abril y mayo de 2015, tanto para los 
docentes como para los estudiantes. La fecha aseguró que los contenidos programáticos o micropedagógico estuvieran adelantados en un 50 por ciento.

- La triangulación de resultados y análisis, se hizo una vez se aplicaron los instrumentos y se logró tener un primer diagnóstico sobre la aplicación de las TIC.

\section{RESULTADOS}

Tabla 1. Categoría y Subcategorías de Análisis de la Entrevista

\begin{tabular}{|c|c|c|c|}
\hline $\begin{array}{c}\text { Categorí } \\
\text { a de } \\
\text { análisis }\end{array}$ & $\begin{array}{c}\text { Sub- } \\
\text { categoría }\end{array}$ & Definición & Descripción \\
\hline $\begin{array}{l}\text { Uso de TIC } \\
\text { en el aula }\end{array}$ & $\begin{array}{l}\text { El uso de las } \\
\text { TIC } \\
\text { desarrolla } \\
\text { investigación } \\
\text { en el aula }\end{array}$ & $\begin{array}{l}\text { La } \\
\text { subcategoría } \\
\text { hace } \\
\text { referencia a } \\
\text { que las TIC } \\
\text { hacen parte } \\
\text { del trabajo } \\
\text { en el aula } \\
\text { especialmen } \\
\text { te para } \\
\text { reforzar } \\
\text { conceptos y } \\
\text { acciones } \\
\text { prácticas. }\end{array}$ & $\begin{array}{l}\text { ENT 1: } \\
\text { "Utilizada para } \\
\text { investigar } \\
\text { términos y } \\
\text { trabajar en los } \\
\text { proyectos de } \\
\text { investigación } \\
\text { cuantitativa". } \\
\text { ENT 2: "Lo } \\
\text { utilizo como } \\
\text { guía } \\
\text { fundamental } \\
\text { para explicar } \\
\text { los procesos de } \\
\text { diseño y el uso } \\
\text { de } \\
\text { herramientas } \\
\text { para la edición } \\
\text { grafica en } \\
\text { software como } \\
\text { Corel Draw y } \\
\text { Adobe". } \\
\text { ENT 3: "No } \\
\text { utilizo el } \\
\text { computador". } \\
\text { ENT 4: } \\
\text { "Investigación } \\
\text { en la web } \\
\text { sobre los } \\
\text { temas de clase, } \\
\text { especialmente } \\
\text { Google } \\
\text { académico". }\end{array}$ \\
\hline
\end{tabular}

\begin{tabular}{|c|c|c|c|}
\hline $\begin{array}{l}\text { Uso del } \\
\text { computad } \\
\text { or en el } \\
\text { aula }\end{array}$ & $\begin{array}{l}\text { Uso del } \\
\text { computador } \\
\text { para el } \\
\text { desarrollo } \\
\text { curricular }\end{array}$ & $\begin{array}{l}\text { Hace } \\
\text { referencia al } \\
\text { uso que se le } \\
\text { da al } \\
\text { computador } \\
\text { como } \\
\text { herramienta } \\
\text { de trabajo } \\
\text { para el } \\
\text { desarrollo } \\
\text { curricular }\end{array}$ & $\begin{array}{l}\text { ENT 1: } \\
\text { "Prácticament } \\
\text { e no se utiliza } \\
\text { hasta el } \\
\text { momento". } \\
\text { ENT 2: "No se } \\
\text { ha } \\
\text { implementado } \\
\text { en el curso, la } \\
\text { implementació } \\
\text { n del } \\
\text { computadoro } \\
\text { cualquier otro } \\
\text { dispositivo } \\
\text { electrónico } \\
\text { hasta el } \\
\text { momento". } \\
\text { ENT 4: "Como } \\
\text { no se está } \\
\text { usando, la } \\
\text { autoevaluació } \\
\text { n no verifica } \\
\text { esta } \\
\text { herramienta". } \\
\text { ENT 2: "En el } \\
\text { momento, de } \\
\text { acuerdo a la } \\
\text { modalidad de } \\
\text { enseñanza del } \\
\text { curso, no veo } \\
\text { de manera } \\
\text { necesaria el } \\
\text { uso del } \\
\text { computador" }\end{array}$ \\
\hline $\begin{array}{l}\text { Uso de } \\
\text { TIC para el } \\
\text { desarrollo } \\
\text { de } \\
\text { competen } \\
\text { cias }\end{array}$ & $\begin{array}{l}\text { Las } \\
\text { competencia } \\
\text { s en TIC }\end{array}$ & $\begin{array}{l}\text { Hace } \\
\text { referencia a } \\
\text { como las } \\
\text { nuevas } \\
\text { tecnologías } \\
\text { permiten } \\
\text { acercarse al } \\
\text { nuevo } \\
\text { conocimient } \\
\text { o y generar } \\
\text { nuevas ideas } \\
\text { entre el } \\
\text { estudiante }\end{array}$ & $\begin{array}{l}\text { ENT 1: "Toma } \\
\text { de decisiones, } \\
\text { trabajo } \\
\text { compartido y } \\
\text { responsabilida } \\
\text { des". } \\
\text { ENT 3: } \\
\text { "Considero } \\
\text { que utilizando } \\
\text { las TIC se } \\
\text { puede explicar } \\
\text { fácilmente } \\
\text { algunas } \\
\text { instrucciones } \\
\text { complejas y } \\
\text { asegurar la } \\
\text { comprensión } \\
\text { de los } \\
\text { estudiantes, } \\
\text { esto hace que } \\
\text { la clase se más } \\
\text { interactiva y } \\
\text { agradable lo } \\
\text { que puede } \\
\text { mejorar la } \\
\text { asistencia del } \\
\text { alumnado } \\
\text { favoreciendo } \\
\text { la } \\
\text { concentración }\end{array}$ \\
\hline
\end{tabular}




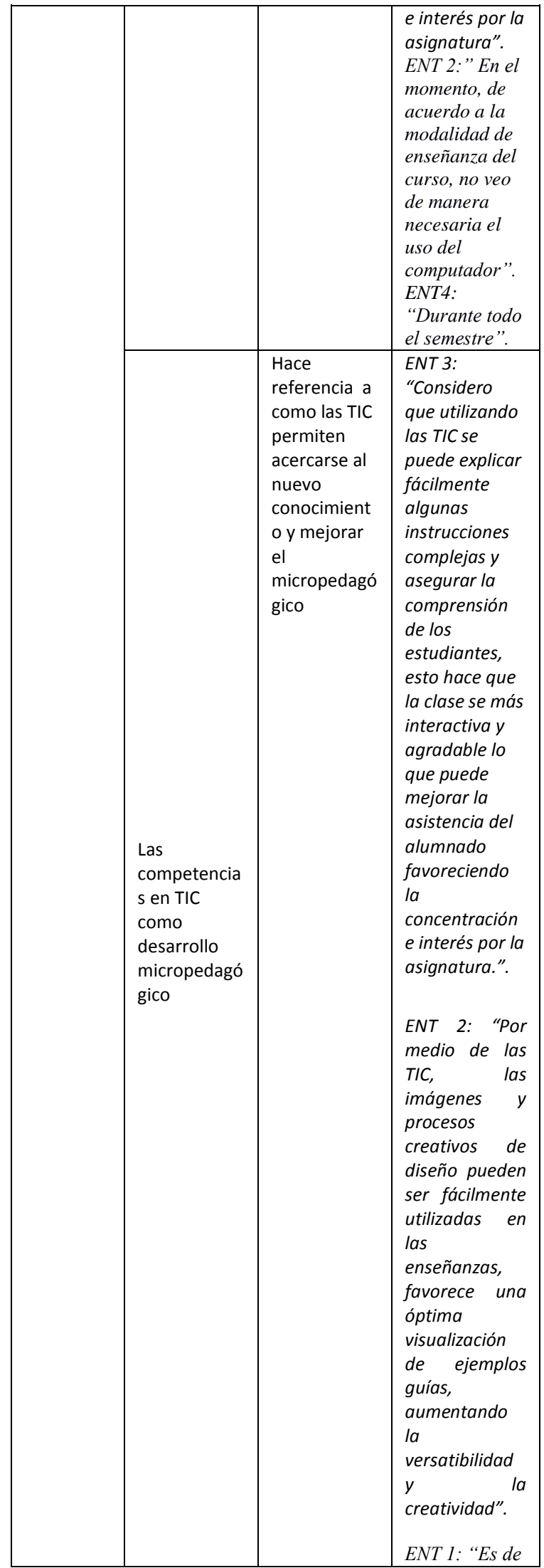

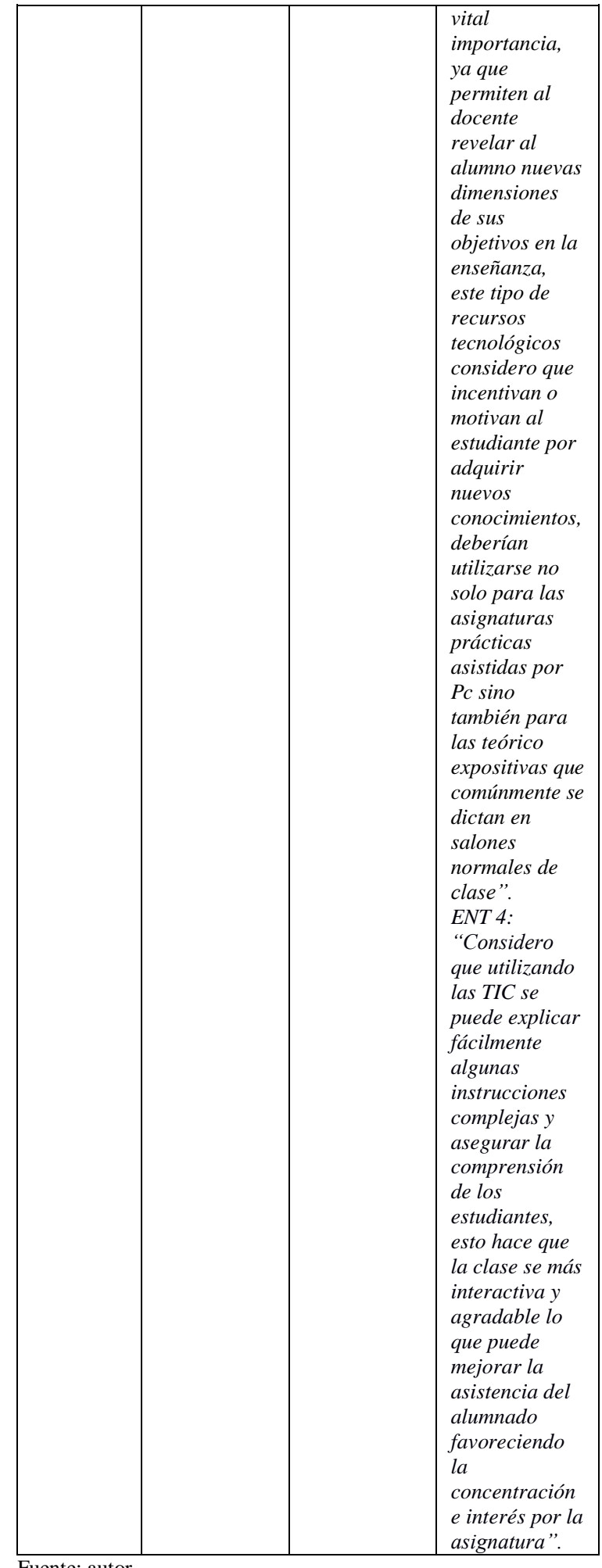




\section{CONCLUSIONES}

En esta sección se mencionan las conclusiones de la investigación muy sucintamente, a modo de recordatorio de las ideas más importantes.

\section{BIBLIOGRAFÍA}

Baquero, R. Carretero, M. Camilloni, A. (1997) El debate constructivista. Argentina, Buenos Aires: Aique.

Castells, M. (2006) La Era de la Información. Vol. I: La Sociedad Red. Distrito Federal, México. EditorialSiglo XXI.

Coll, C (Coord.). (2008). Desarrollo, Aprendizaje y Enseñanza en la Educación Secundaria. España: GRAO.

Consejo Superior Universitario, Acuerdo No.006, marzo 05 de 2003.

Díaz Quero, V. (2006). Formación docente, práctica pedagógica y saber pedagógico. Laurus, 12(Ext) 88-103. Recuperado de: from http://www.redalyc.org/articulo.oa?i $\underline{\mathrm{d}=76109906}$ proyecto de las universidades. Revista Iberoamericana de Educación, 35(1), 151-164.

Guevara, F. D. (2012). Evaluation per competences within the currículum in higher education. Panorama, 6(10), 133-151.

Hernández, S., Fernández, C., \& Baptista, L. (2003). Metodología de la Investigación. TI y

II. Editorial Félix Varela, La Habana.

Llinás, R. (2000). El reto: educación, ciencia y tecnología. Tercer Mundo Editores.

García-Cabrero, B., Loredo, J., Luna, E., Rueda, M. (2008). Modelo de evaluación de competencias docentes para educación media y media superior. Revista Iberoamericana de Evaluación Educativa 1(3ed), 124-136. Recuperado de:http://www.rinace.net/riee/numeros/vol 1-num3_e/art8.pdf

González, J., Wagenaar, R., \& Beneitone, P. (2004). Tuning-América Latina: un 